\title{
Endemic asthma inhaler abuse among antisocial adolescents
}

\author{
Brian E. Perron ${ }^{\mathrm{a}, *}$, Matthew O. Howard ${ }^{\mathrm{b}}$ \\ ${ }^{a}$ University of Michigan, School of Social Work, 1080 South University Avenue, Ann Arbor, MI 48109, United States \\ ${ }^{\mathrm{b}}$ University of North Carolina at Chapel Hill, School of Social Work, Tate-Turner-Kuralt Building, 325 Pittsboro Street, \\ CB\# 3550 Chapel Hill, NC 27599-3550, United States
}

Received 11 November 2007; received in revised form 11 January 2008; accepted 11 January 2008

Available online 14 March 2008

\begin{abstract}
Background: Approximately 40 million prescriptions were dispensed in the U.S. for asthma inhalers in 2006. Although the pervasive distribution of asthma inhalers warrants greater attention to possible misuse of these products, few investigations have examined asthma inhaler misuse. The purpose of this study was to describe the prevalence, correlates and consequences of asthma inhaler misuse among antisocial youth.

Method: A cross-sectional survey with face-to-face interviews assessing substance use, psychiatric symptoms, and antisocial behaviors among adolescents $(N=723)$ in residential treatment.

Results: More than 26\% (N=193) of youth were diagnosed with asthma; $91.2 \%$ of asthmatic youth had received a prescription for an inhaler. Of the 373 youth who had used a prescribed or non-prescribed asthma inhaler, $23.6 \%(N=88)$ reported using an inhaler to get high (i.e., were inhaler misusers). Asthma inhaler misusers had an earlier onset of antisocial conduct, significantly greater levels of current psychiatric distress and lifetime suicidality, higher rates of lifetime substance use problems and volatile solvent abuse, and significantly higher levels of temperamental impulsivity and fearlessness than did inhaler non-users or users. Caucasian racial status, current level of psychiatric distress, and lifetime volatile solvent abuse significantly distinguished lifetime asthma inhaler users and misusers in multivariate logistic regression analyses. Asthma inhaler misusers were significantly more likely to report euphoria, memory problems, slurred speech, blurred vision, confusion, dizziness, and a variety of other acute reactions to asthma inhaler use than were asthma inhaler users.

Conclusions: Asthma inhaler misuse for the purposes of getting high was prevalent among antisocial youth, co-occurred with other psychiatric and substance use problems, and was associated with adverse consequences.
\end{abstract}

(C) 2008 Elsevier Ireland Ltd. All rights reserved.

Keywords: Asthma inhalers; Asthma; Antisocial use; Inhalants; Volatile solvents

\section{Introduction}

Approximately 20 million U.S. residents suffer from asthma, an often chronic lung condition leading to nearly 500,000 hospitalizations and more than 4000 deaths annually (Akinbami, 2007). For most of the past quarter century, the prevalence and incidence of asthma have increased annually (Mannino et al., 2002; Rudd and Moorman, 2007), such that approximately $8 \%$ of the general U.S. population is currently afflicted with the disorder (Akinbami, 2007).

In 2006, nearly 40 million prescriptions for asthma inhalers were dispensed and revenue from the sale of relief inhalers (i.e., those used to treat acute asthma attacks) approached half-a-

\footnotetext{
* Corresponding author. Tel.: +1 734763 9272; fax: +1 7347633372.

E-mail address: beperron@umich.edu (B.E. Perron).
}

billion dollars (IMS Intelligence, 2007). In excess of 29 million prescriptions were dispensed for albuterol inhalers alone in 2006, making albuterol the 10th most dispensed pharmaceutical product in the U.S. that year (IMS Intelligence, 2007).

Although the pervasive distribution of asthma inhalers in the U.S. and elsewhere warrants greater attention to possible misuse of these products, few investigations have examined the prevalence, patterns, correlates, or consequences of asthma inhaler misuse. Most studies heretofore published consist of case reports. For example, Edwards and Holgate (1979) described the case of a 24-year-old man who self-administered between 60 and 90 daily doses of a salbutamol (i.e., the International Nonproprietary Name for albuterol, a $\beta_{2}$-adrenergic receptor agonist), inhaler noting that "he became dependent upon the inhalers and tolerant of their effects and behaved in a way more reminiscent of a drug abuser than that of a man distressed by asthma. He frequently presented himself to his general practitioner saying 
that he had lost his prescription and requesting a repeat, while showing minimal signs of bronchospasm" (p. 624).

Pratt (1982) presented two cases of salbutamol inhaler abuse. Grand mal seizures associated with inhaler abuse were observed in a 15-year-old girl and it was noted that "she had used twenty inhalers in the previous year often with excessive daily use, and was then persistently vociferous demanding salbutamol" (p. 205). In the case of a 15-year-old boy, poor appetite, fatigue, generalized disinterest, and false reports minimizing extent of inhaler use were noted. Pratt (1982) observed, as have other scholars in this area, that salbutamol and steroid asthma inhalers contained fluorinated hydrocarbon propellants, making it unclear whether it was the salbutamol or propellant hydrocarbons on which youth had become dependent. In this vein, Pratt (1982) remarked that "no case of steroid inhaler dependence seems to be recorded" (p. 208), raising the possibility that it was the salbutamol (albuterol) and not the propellants on which youth had become dependent.

Prasher and Corbett (1990) presented a case and reviewed 12 additional cases of salbutamol inhaler dependence reported in eight earlier studies (Gluckman, 1974; Gaultier et al., 1976; Kjellman, 1977; Brennan, 1983; Thompson et al., 1983; Raine, 1984; Slessor, 1984; O’Callaghan and Milner, 1988). Effects of inhaler misuse described in these studies included aggression, acute psychosis, and use of inhalers to "self-medicate" anxious or dysphoric mood states. More recently, case studies of albuterol inhaler abuse by children have been reported in the U.S. and associated with hypokalemia, hallucinations, and other deleterious health and behavioral conditions (Schnapf and Santeiro, 1994; Rakhmanina et al., 1998).

Boyd et al. (2004, 2006) reported the only efforts of which we are aware to examine the prevalence and correlates of asthma inhaler misuse. Boyd et al. (2004) surveyed 1723, 6th-11th graders in one school district in the Detroit, Michigan area in 2002 . Overall, nearly $11 \%$ of youth (15\% of 8 th/9th graders) reported lifetime use of "someone else's asthma inhaler." Few demographic factors distinguished inhaler misusers and nonmisusers. Significantly larger proportions of youth who had used someone else's asthma inhaler had smoked cigarettes or used alcohol in the preceding month and used alcohol, marijuana, "ecstasy" or cocaine in the prior year, compared to non-misusers of asthma inhalers. Neither the frequency of nor motives for non-prescribed asthma inhaler misuse were assessed. Further, youth were not asked whether they had received a formal asthma diagnosis from a physician or other health care provider or if they had been prescribed asthma inhaler medication. Psychiatric and psychosocial correlates of asthma inhaler misuse were not examined.

Boyd et al. (2006) conducted a similar survey of 1017, 5th-10th graders in 2003. Inhaler misuse was defined as any use of an asthma inhaler for which the youth did not have a prescription. Four groups of youths were identified in relation to asthma inhaler use and misuse: (1) inhaler non-users (youth who had never had a prescription for an inhaler and who reported never misusing one); (2) inhaler users (youth who had received a prescription for an inhaler and who reported never having misused an inhaler; (3) inhaler users/misusers (youth who had received a prescription for an asthma inhaler and who reported use of an inhaler for which they did not have a prescription); and (4) inhaler misusers (youth who had never received a prescription for an asthma inhaler and who reported use of an inhaler for which they did not have a prescription).

Few significant demographic differences between groups of asthma inhaler non-users, users, and misusers were identified. In general, both groups of inhaler misusers had significantly higher rates of monthly cigarette, alcohol, and marijuana use, and annual illicit drug use than inhaler non-users and users. Some tendency was noted for the group of inhaler misusers to show the highest levels of substance use. Logistic regression analyses indicated that few factors were associated with lifetime or past year inhaler misuse, except for having received a lifetime prescription for an asthma inhaler, which was strongly associated with lifetime and past year inhaler misuse (odds ratios $(\mathrm{OR})=8.0$ and 7.2, respectively)

Presently, the specific effects motivating adolescent inhaler misuse for the purposes of intoxication are unclear. Recent reports have documented significantly elevated rates of depression, posttraumatic stress disorder, and other anxiety disorders in persons with asthma (Goodwin et al., 2007). For example, Katon et al. (2007) found that youth 11-17 with asthma had a twofold increase in risk for anxiety or depressive disorders compared to control youth. Girls, socioeconomically disadvantaged youth, and youth with relatively recent onset of asthma were at especially high risk for comorbid asthma and psychiatric disorders. Thus, it is conceivable that asthmatic youth may be self-medicating comorbid psychiatric disorders and that intoxication may be a key determinant or "side effect" of such self-medicating inhaler misuse. It is currently unclear whether psychiatric disorders enhance risk for asthma, asthma raises risk for psychiatric disorders, or both disorders share common risk factors (Kuehn, 2008).

With regard to pharmacological actions, albuterol inhalers are known to increase heart rate and blood pressure and there are anecdotal reports of inhaler misuse for their stimulatory effects (Martin et al., 1995) and for the purpose of weight loss (American Academy of Allergy, Asthma, and Immunology, 2006).

Antisocial adolescents are a nearly ideal population in which to study asthma inhaler misuse and abuse. Not only do they constitute a large and important group in their own regard, such youth also represent an enriched population for the study of highrisk behaviors such as substance abuse. Findings from studies of antisocial youth can also inform investigations of general population youth samples. For example, Howard and Jenson (1999) found that one-third of the state population of juvenile probationers they studied had abused volatile solvent inhalants such as gasoline and glue. More recent investigations, such as that reported by $\mathrm{Wu}$ et al. (2004) have extended these findings to the general U.S. population of 13-17-year-old, noting similar patterns and associations with use, but reporting a lifetime prevalence rate of volatile solvent inhalation of approximately $9 \%$.

Given the widespread diffusion of asthma inhalers in the U.S. and many other nations, conspicuous dearth of current informa- 
tion regarding the etiology, epidemiology, and consequences of asthma inhaler misuse, and potential public health and clinical importance of these findings, this investigation (1) described the prevalence and patterns of asthma inhaler misuse in a state population of antisocial youth known to be at high risk for substance abuse generally and abuse of volatile solvent inhalants specifically (Howard et al., 2007; Howard and Jenson, 1999; Wu et al., 2004); (2) identified factors associated with asthma inhaler misuse; and (3) examined the prevalence of various physical and psychological reactions to acute asthma inhaler use/misuse across groups of inhaler users and misusers.

\section{Methods}

\subsection{Sample}

Findings of the present study are based on a survey conducted in 2003 of the population of current residents of the Missouri Division of Youth Services (DYS). The Missouri DYS is the legal guardian of all residents who are committed to its care by the state's 45 juvenile courts. Ten adolescents were on furlough at the time of interviewing and 2 adolescents were transferred to another DYS facility while interviewers were at the facility, but before they could be interviewed. Of the 728 adolescents available to interview, all agreed to participate. However, 5 interviews were discontinued; 4 adolescents displayed signs or reported symptoms of psychosis and one adolescent chose not to continue. The 723 adolescents who completed the interview constituted $97.7 \%$ of DYS residents at the time interviewing was conducted, $99.3 \%$ of residents available for interviewing, and approximately $55.0 \%$ of adolescents committed to DYS care in the prior year. Thus, the present study is virtually a census of the population of DYS residents at the time the study was undertaken and a large, representative sample of DYS annual residents.

Participation in the study was voluntary. Face-to-face interviews of adolescents were conducted using a comprehensive assessment inventory. All interviewers completed an intensive 1-day training session and an interview editor was on-site at each facility as adolescents were interviewed to minimize interviewer omissions and errors. DYS residents are under constant observation; thus, interviews were conducted in large rooms that provided private areas where individual confidential interviews could be conducted simultaneously with between 3 and 6 adolescents.

The sample recruitment protocol ensured that no adolescent who had completed the interview at one DYS facility subsequently completed the interview at another DYS facility. This study was approved by the DYS Institutional Review Board, Washington University Human Studies Committee Institutional Review Board, federal Office of Human Research Protection, and was granted a Certificate of Confidentiality by the National Institute on Drug Abuse (NIDA). Adolescents received $\$ 10.00$ for their participation.

All adolescents who provided informed assent completed the Volatile Solvent Screening Inventory (VSSI), a structured face-to-face interview. The VSSI takes approximately $45 \mathrm{~min}$ to complete and assesses demographic characteristics, medical history, lifetime/annual use of 55 volatile solvent inhalants, other drug use and substance-related problems, current psychiatric symptoms, suicidality, trauma history, antisocial traits and criminal activity. A detailed description and copy of the VSSI is available in Howard et al. (2007).

\subsection{Measures}

2.2.1. Asthma diagnosis, inhaler use, and inhaler misuse. Youth were asked to indicate (yes or no) whether they had "ever been diagnosed with asthma by a doctor or nurse." Youth who reported having been diagnosed with asthma, were then asked to indicate (yes or no) whether they "were ever prescribed an inhaler to help treat your asthma?" Youth who reported having been prescribed an inhaler were then asked to respond (yes or no) to the question, "Did you ever use your asthma inhaler more than you were supposed to according to the doctor's or pharmacist's instructions?" Respondents who reported using their prescribed asthma inhaler more than they were supposed to according to the doctor's or pharmacist's instruction were asked about three potential reasons for their misuse. Possible reasons for youths' use of a prescribed asthma inhaler more than they were supposed to included use because they were "treating their own asthma symptoms," "wanted to see what it would feel like," or "were trying to get high."

All youth were also asked whether they had ever used an asthma inhaler for which they did not have a prescription. Study participants who had used an asthma inhaler for which they did not have a prescription were asked to indicate reasons why they had used a non-prescribed inhaler. Reasons included "treating their own asthma symptoms," "wanted to see what it would feel like," or "were trying to get high."

These survey questions were used to classify subjects into one of three groups. Inhaler non-users were youth without any history of asthma inhaler use. Inhaler users were youth who reported any use of prescription and/or nonprescription asthma inhalers for purposes other than to get high. Thus, inhaler users were youth who used asthma inhalers as prescribed or who used prescribed asthma inhalers at greater than prescribed levels or non-prescribed inhalers to medicate their own asthma symptoms or to see what it would feel like, but never for the purposes of getting high. Inhaler misusers were youth who reported any prior use of prescribed or non-prescribed asthma inhalers to get high. Although there are many potential criteria by which asthma inhaler groups could be formed, our method has the virtue of establishing clearly defined groups of inhaler non-users and misusers. The inhaler user group includes a more heterogeneous assemblage of inhaler users than the other two groups; however, the inclusion of youth who used prescribed inhalers at levels higher than prescribed or non-prescribed inhalers because they wanted to see what it would feel like in this group suggests that the inhaler user vs. misuser contrasts reported below are conservative in nature.

2.2.2. Physical and psychological responses to inhalers. Inhaler users and misusers were asked how frequently they had experienced each of 25 different physical and psychological responses (e.g., euphoria, dizziness, anxiety) while using or immediately following use of an asthma inhaler. Response options for each item were: $0=$ Never, $1=$ Rarely, $2=$ Occasionally, $3=$ Frequently, and $4=$ Always. As the distributions of responses were highly skewed, responses were dichotomized for each item to reflect the relative absence ("Never" or "Rarely" response options) vs. presence ("Occasionally," "Frequently," or "Always" response options) of each of the 25 responses to asthma inhaler use/misuse.

2.2.3. Demographic and psychosocial variables. Gender, age, self-reported racial status, grade (current or last completed), family receipt of public assistance, and geographical area of family residence (i.e., urban, suburban, small town, rural) were recorded for each youth. Youth were also asked at what age they committed their first crime.

2.2.4. Substance use and related problems. Subjects were queried about their ages at first use of alcohol and marijuana, respectively. For subjects who had used alcohol and marijuana, the lower of the two ages was selected. Respondents who reported any use of one or more of 55 volatile solvent inhalants with the expressed intention of getting high were considered lifetime volatile solvent users (see Howard et al., 2007 supplementary material for a listing of volatile solvent inhalants assessed).

Lifetime substance-related problems were assessed with the 8-item Alcohol/Drug Use Scale of the Massachusetts Adolescents Screening Instrument-2nd Version (MAYSI-2) (Grisso and Barnum, 2000) developed for use with juvenile justice populations. Adolescents responded "yes" or "no" to questions about whether they had ever been drunk or high at school, had used alcohol and drugs at the same time, had ever been so drunk or high they could not remember what happened, used alcohol or drugs to help them feel better, had gotten into trouble while high or drinking (if yes) whether or not that trouble had been fighting, had done anything they wished they had not while drunk or high, or had their parents think they drink too much. Scores could range from 0 to 8 . Grisso and Barnum (2000) found the scale to be internally consistent $(\alpha=.86)$ in their norming sample; the $\alpha$ coefficient in this study was .83 . 
2.2.5. Psychiatric variables. All respondents completed the Brief Symptom Inventory (BSI), consisting of 53 items assessing the extent to which adolescents were "bothered or disturbed" ( $0=$ not at all; $4=$ extremely) by a variety of thoughts or feelings "over the last 7 days including today" (Derogatis, 1993). The BSI yields a Global Severity Index of overall current psychiatric distress (possible range $=0-212, \alpha=.96$ in current study).

Adolescents completed the 5-item MAYSI-2 suicide ideation scale, which requires adolescents to respond "yes" or "no" to questions assessing whether or not they have ever wished they were dead, have felt like life was not worth living, have felt like hurting themselves, have felt like killing themselves, and have ever given up hope for their life. Grisso and Barnum (2000) reported a $\alpha$ coefficient of .83 ; the $\alpha$ coefficient in this study was .91 .

Study participants also completed the 56-item Psychopathic Personality Inventory Short-Version (PPI-SV) (Lilienfeld and Andrews, 1996). Youth were asked to decide to what extent each of the personality characteristics described in each statement were false or true as applied to them $(1=$ false, $2=$ mostly false, $3=$ mostly true, $4=$ true). Two subscales from the PPI-SV were included in this study: fearlessness and impulsive nonconformity. Both measures had a possible range of 0-28. Alpha coefficients for these measures in this study were .68 and .55 , respectively.

\subsection{Analytic procedures}

Descriptive statistics were used to summarize study results. Pearson's chi-square $\left(\chi^{2}\right)$ tests, Fisher's exact tests, and one-way analysis of variance (ANOVA) were used to examine bivariate associations. Associations were also summarized as effect sizes using unadjusted odds ratios with $95 \%$ confidence intervals, Cramer's $V(V)$, and $R$-squared $\left(R^{2}\right)$ for chi-square and ANOVA tests. Multivariate logistic regression analysis was used to identify variables associated with inhaler misuse. A small set of subjects did not respond to questions about psychological and physical consequences to inhalants. Listwise deletion was used for these analyses. Less than $1 \%$ of the data points for the independent variables were missing, which were imputed using the aregImpute function in the HMisc package for $R$ (Harrell, 2002).

\section{Results}

Table 1 provides a summary of sample characteristics. Over half the study sample was White and approximately one-third $(32.9 \%, n=238)$ was African American. Only 3.9\% of the sample $(n=28)$ was Latino/Latina, $6.2 \%(n=45$, ) biracial, and $1.5 \%$ $(n=11)$ of another race. Eighty-seven percent of youth were male, and ages ranged from 11 to 20 (mean=15.5, S.D. = 1.2). Due to the relatively small number of Latino/Latina and biracial adolescents and adolescents of another race, the race/ethnicity variable was dichotomized for certain bivariate and multivariate analyses ("White" vs. "non-White"). Approximately forty percent of participants reported that their family received public assistance. Prior to being incarcerated, the majority of adolescents lived in small towns $(39.5 \%, n=286)$ or urban cities $(39.1 \%, n=283)$. Smaller percentages lived in suburban $(13.8 \%, n=100)$ or rural areas $(7.5 \%, n=54)$. This variable

Table 1

Comparison of asthma inhaler non-users, users, and misusers across demographic, psychosocial, and clinical measures

\begin{tabular}{|c|c|c|c|c|c|}
\hline \multirow[t]{2}{*}{ Variables } & \multirow[t]{2}{*}{ Full sample $(N=723)$} & \multicolumn{4}{|c|}{ Subgroup comparison } \\
\hline & & $\begin{array}{l}\text { Inhaler non-users } \\
(N=350)\end{array}$ & $\begin{array}{l}\text { Inhaler users } \\
(N=285)\end{array}$ & $\begin{array}{l}\text { Inhaler misusers } \\
(n=88)\end{array}$ & Results \\
\hline Male & $629(87.0)$ & $306(87.4)$ & $254(89.1)$ & $69(78.4)$ & $\chi^{2}(2)=6.18$ \\
\hline Female & $94(13.0)$ & $44(12.6)$ & $31(10.9)$ & $19(21.6)$ & $p=\mathbf{0 3}$, Cramer's $V=.10$ \\
\hline \multicolumn{6}{|l|}{ Ethnicity: $N(\%)$} \\
\hline Age: mean (S.D.) & $15.5(1.2)$ & $15.5(1.19)$ & $15.4(1.3)$ & $15.7(1.1)$ & $F(2,720)=1.88, p=.15, R^{2}=.01$ \\
\hline \multicolumn{6}{|l|}{ Urbanicity: $N(\%)$} \\
\hline Rural/small town & $340(47.0)$ & $171(48.9)$ & $125(43.9)$ & $44(50.0)$ & $\chi^{2}(2)=1.93$ \\
\hline Urban/suburban & $383(53.0)$ & $179(51.1)$ & $160(56.1)$ & $44(50.0)$ & $p=.40$, Cramer's $V=.05$ \\
\hline Global severity index sum ${ }^{\mathrm{a}}$ & $3.45(.9)$ & $3.31(.9)$ & $3.44(1.0)$ & $4.03(.7)$ & $F(2,720)=21.29^{\mathrm{b}, \mathrm{c}}, \boldsymbol{p}=\mathbf{. 0 0}, R^{2}=.06$ \\
\hline Suicide ideation ${ }^{\mathrm{a}}$ : mean (S.D.) & $2.21(2.4)$ & $1.85(2.2)$ & $2.20(2.4)$ & $3.73(2.4)$ & $F(2,720)=23.58^{\mathrm{b}, \mathrm{c}}, \boldsymbol{p}=\mathbf{. 0 0}, R^{2}=.06$ \\
\hline $\begin{array}{l}\text { Impulsive non-conformity: } \\
\text { mean (S.D.) }\end{array}$ & $14.81(4.1)$ & $14.52(3.9)$ & $14.65(4.1)$ & $16.48(4.6)$ & $F(2,720)=8.51^{\mathrm{b}, \mathrm{c}}, \boldsymbol{p}=\mathbf{. 0 0}, R^{2}=.02$ \\
\hline Fearlessness: mean (S.D.) & $17.10(5.2)$ & $16.56(5.2)$ & $17.04(5.2)$ & $19.42(5.1)$ & $F(2,720)=10.75^{\mathrm{b}, \mathrm{c}}, \boldsymbol{p}=\mathbf{. 0 0}, R^{2}=.03$ \\
\hline $\begin{array}{l}\text { Substance use problems index: } \\
\text { mean (S.D.) }\end{array}$ & $3.85(2.4)$ & $3.39(2.4)$ & $3.86(2.4)$ & $5.67(1.9)$ & $F(2,720)=33.92^{\mathrm{a}, \mathrm{b}, \mathrm{c}}, \boldsymbol{p}=\mathbf{. 0 0}, R^{2}=.09$ \\
\hline \multicolumn{6}{|c|}{ Lifetime use of other volatile solvent inhalants: $N(\%)$} \\
\hline No & $444(61.4)$ & $250(71.4)$ & $184(64.6)$ & $10(11.4)$ & $\chi^{2}(2)=112.63$ \\
\hline Yes & $279(38.6)$ & $100(28.6)$ & $101(35.4)$ & $78(88.6)$ & $\boldsymbol{p}=\mathbf{. 0 0}$, Cramer's $V=.39$ \\
\hline
\end{tabular}

Note: All percentages are reported as column percentages. Values in bold are significant $(p<.05)$. All contrasts for one-way analysis of variance were tested using Tukey's post-hoc contrast test at $p<.05$. Significant differences in contrasts are noted by the following superscripts: a = non-users significantly different than users; $\mathrm{b}=$ non-users significantly different than misusers; and $\mathrm{c}=$ users significantly different than misusers.

${ }^{\text {a }}$ Values are log-transformed due to skewness. 
was dichotomized into urban/suburban vs. rural/small town categories. Scores on the Brief Symptom Inventory Global Severity Index ranged from 0 to 172 (mean $=43.8$, median $=36.0$, S.D. $=34.8$ ). Given the excess skew of the Global Severity Index score distribution (skew $=1.20$ ), a log transformation of this variable was used (skew $=-.83$ ) in subsequent analyses. It is notable that females (21.6\% vs. $10.9 \%)$ and whites $(76.1 \%$ vs. $47.7 \%)$ were substantially overrepresented in inhalant misuser group compared to the inhaler user group.

\subsection{Prevalence of asthma and asthma inhaler prescriptions}

More than 26\% $(n=193)$ of the overall sample reported a lifetime diagnosis with asthma. Ethnicity was significantly ( $p=.006)$ associated with a lifetime asthma diagnosis, with Biracial and African Americans having the highest rates (42.2\% and $31.1 \%$, respectively), followed by Caucasians $(24.1 \%)$, Latino/Latinas (10.7\%), and youth of other races (9.1\%). Subsequent analyses identified no significant differences in asthma prevalence rates between White and African American or between White and non-White youth. Receipt of a lifetime asthma diagnosis was not significantly associated with age, family receipt of public assistance, or geographic place of residence prior to incarceration. Among subjects with a lifetime asthma diagnosis, $91.2 \%(n=176)$ had been prescribed an asthma inhaler. No significant associations among demographic variables and receipt of an asthma inhaler prescription were identified.

\subsection{Patterns of inhaler use and misuse}

3.2.1. Prescribed inhaler use and misuse. Among youth who had received a prescription for an asthma inhaler, $26.7 \%(n=47)$ reported lifetime use of their inhaler at greater than prescribed levels. Of these 47 persons, approximately half $(48.9 \%, n=23)$ indicated that they were using at greater than prescribed levels to treat their own asthma symptoms; $59.6 \%(n=28)$ wanted to see what it would feel like; $57.4 \%(n=27)$ reported that they were trying to get high. None of the demographic or clinical variables assessed were significantly associated with the different reasons for prescription inhaler misuse based on a series of chi-square tests.

3.2.2. Non-prescribed inhaler use and misuse. Approximately one-third $(33.1 \%, n=239)$ of the overall sample reported having used an asthma inhaler for which they did not have a prescription. Approximately 9\% $(n=22)$ of these youth had used a non-prescribed asthma inhaler, even though they reported having received a prior prescription for an asthma inhaler. Reasons for using an asthma inhaler without a prescription included treating one's own asthma symptoms $(33.6 \%, n=80)$; wanting to see what it would feel like to use an inhaler $(65.7 \% ; n=157)$; and wanting to get high $(30.1 \% ; n=72)$.

\subsection{Comparison of inhaler non-users, users, and misusers}

3.3.1. Bivariate associations. As summarized in Table 1, there were no demographic differences between inhaler non-users $(N=350)$, users $(N=285)$, and misusers $(N=88)$. Groups differed significantly with regard to age at first crime, psychiatric (i.e., suicidal ideation; GSI summary score) and substance use problems, and temperamental impulsivity and fearlessness. Effect sizes were small $\left(R^{2}\right.$ values $\left.\leq .09\right)$. The largest difference was for prevalence of lifetime use of volatile solvent inhalants, with the inhaler misuser group exhibiting the highest rate. The effect size for this relationship was significantly larger than the other observed effect sizes $(V=.39)$.

Table 2

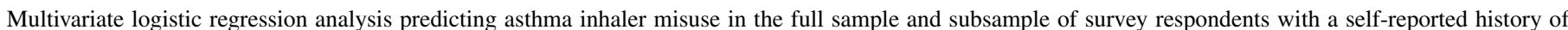
asthma inhaler use

\begin{tabular}{|c|c|c|c|c|}
\hline \multirow[t]{2}{*}{ Variables } & \multicolumn{2}{|c|}{ Full sample $(N=723)$} & \multicolumn{2}{|c|}{ Lifetime history of asthma inhaler use $(N=373)$} \\
\hline & $\begin{array}{l}\text { Adjusted } \\
\text { odds ratio }\end{array}$ & $\begin{array}{l}95 \% \text { confidence } \\
\text { interval }\end{array}$ & $\begin{array}{l}\text { Adjusted odds } \\
\text { ratio }\end{array}$ & $\begin{array}{l}95 \% \text { confidence } \\
\text { interval }\end{array}$ \\
\hline Prior receipt of asthma inhaler prescription (yes $=1$, no $=0$ ) & 2.84 & $1.61-5.04$ & .97 & $.53-1.78$ \\
\hline Gender $($ male $=1$, female $=0)$ & 1.59 & $.76-3.31$ & 1.77 & $.77-4.07$ \\
\hline Ethnicity (White = 1 non-White $=0$ ) & 1.79 & $.95-3.36$ & 2.13 & $1.07-4.24$ \\
\hline Age $(11-20)$ & 1.22 & $.96-1.56$ & 1.30 & $1.00-1.69$ \\
\hline Urbanicity (urban/suburban = 1, rural/small town =0) & 1.62 & $.93-2.84$ & 1.54 & $.80-2.94$ \\
\hline Public assistance (yes) & .77 & $.45-1.34$ & .85 & $.46-1.55$ \\
\hline Age at first crime $(5-16)$ & .92 & $.84-1.01$ & .92 & $.83-1.03$ \\
\hline Global severity index $\left(2.57-5.08^{\mathrm{a}}\right)$ & 2.14 & $1.41-3.25$ & 1.86 & $1.18-2.91$ \\
\hline Suicidal ideation $(0-6)$ & 1.04 & $.92-1.18$ & 1.02 & $.89-1.17$ \\
\hline Impulsivity (7-28) & .99 & $.93-1.05$ & 1.01 & $.94-1.08$ \\
\hline Fearlessness (7-28) & 1.01 & $.95-1.07$ & 1.02 & $.96-1.09$ \\
\hline Substance use problems scale $(0-8)$ & 1.12 & $.97-1.30$ & 1.09 & $.93-1.28$ \\
\hline Lifetime use of volatile solvent inhalants $(1=$ yes, $0=$ no $)$ & 10.45 & $4.78-22.83$ & 9.17 & $4.10-20.49$ \\
\hline
\end{tabular}

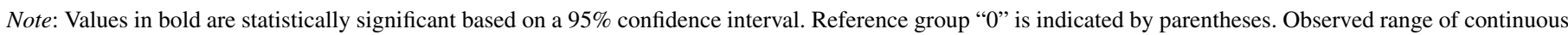
variables is also noted in parentheses.

a Values are log-transformed due to skewness. 
3.3.2. Multivariate associations. Two logistic regression models were specified to identify correlates of inhaler misuse (i.e., using a prescribed or non-prescribed inhaler to get high). The first model included the full sample. This model exhibited a good fit to the data (likelihood ratio $\chi^{2}[13]=162.5, p=.000$, pseudo- $R^{2}=.39$ ). Table 2 summarizes the adjusted OR with $95 \%$ confidence intervals. Youth who received an inhaler prescription were almost three times more likely to be an asthma inhaler misuser than youth without a prescription $(\mathrm{OR}=2.84$, $95 \% \mathrm{CI}=1.61-5.04)$. Youth with a history of volatile solvent inhalant use were over 10 times more likely to be asthma inhaler misusers compared to youth without any prior history of volatile solvent inhalant use $(\mathrm{OR}=10.45,95 \% \mathrm{CI}=4.78-22.83)$. Global severity index score (logged) was also a significant risk factor $(95 \% \mathrm{CI}=2.14,95 \% \mathrm{CI}=1.41-3.25)$. A second model was specified to identify correlates of inhaler use among youth with a lifetime history of asthma inhaler use (i.e., excluding inhaler non-users). Having received an inhaler prescription was not a significant risk factor, but being White (compared to non-White) was statistically significant ( $O R=2.13,95 \%$ $\mathrm{CI}=1.07-4.24)$. The global severity index score (logged) and lifetime use of volatile solvent inhalants were also significant, with effect sizes slightly lower in comparison to the full sample model.

\subsection{Psychological and physical responses}

Table 3 provides overall and subgroup prevalence rates for 25 self-reported acute psychological and physical reactions to asthma inhaler use, ordered by the frequency with which such responses were reported in the overall sample. The most common acute responses to inhaler use among all inhaler users and misusers included feeling more relaxed (26.3\%), feeling dizzy $(25.7 \%)$, and having a headache $(23.7 \%)$. A series of subgroup comparisons were conducted to determine whether the responses to inhalers were significantly different among inhaler users and misusers. Of the 25 comparisons, inhaler misusers were significantly more likely to report all psychological and physical responses to asthma inhalers. In addition to being statistically significant, it is important to highlight the large effect sizes summarized by the unadjusted odds ratios and $95 \%$ confidence intervals (four effect sizes were not computed due to low cell counts $<5$ ). Inhaler misusers were over 33 times more likely to report euphoric feelings

Table 3

Prevalence of self-reported acute physical and psychological responses to asthma inhaler use, overall and across inhaler user and misuser groups

\begin{tabular}{|c|c|c|c|c|}
\hline \multirow[t]{2}{*}{ Responses } & \multirow{2}{*}{$\begin{array}{l}\text { Any inhaler use }{ }^{\mathrm{a}} \text {, } \\
N(\%)\end{array}$} & \multicolumn{3}{|l|}{ Subgroup comparisons } \\
\hline & & Inhaler users, $N(\%)$ & Inhaler misusers, $N(\%)$ & $\operatorname{UOR}(95 \% \mathrm{CI})^{\mathrm{b}}$ \\
\hline Feel more relaxed & $98(27.4)$ & $63(23.2)$ & $35(40.2)$ & $2.22(1.33-3.71)$ \\
\hline Feel dizzy & $92(25.7)$ & $38(14.0)$ & $54(62.1)$ & $\mathbf{1 0 . 0 3}(5.78-17.43)$ \\
\hline Have a headache & $85(23.7)$ & $44(16.2)$ & $41(47.1)$ & $4.60(2.71-7.82)$ \\
\hline Have a rapid heartbeat ${ }^{c}$ & $64(17.9)$ & $27(10.0)$ & $37(43.0)$ & $\mathbf{6 . 8 2}(3.81-12.23)$ \\
\hline Feel more tired or fatigued than normal & $60(16.8)$ & $31(11.4)$ & $29(33.3)$ & $3.87(2.16-6.93)$ \\
\hline Feel more anxious & $59(16.5)$ & $20(7.4)$ & $39(45.3)$ & $\mathbf{1 0 . 4 1}(5.59-19.41)$ \\
\hline Feel more talkative & $56(15.6)$ & $23(8.5)$ & $33(37.9)$ & $6.59(3.59-12.11)$ \\
\hline Feel euphoric (feeling “high,” happy, carefree) & $52(14.5)$ & $8(3.0)$ & $44(50.6)$ & $33.64(14.82-76.33)$ \\
\hline Feel panicky ${ }^{\mathrm{c}}$ & $43(12.0)$ & $14(5.2)$ & $29(33.3)$ & $9.14(4.55-18.39)$ \\
\hline Feel more powerful or confident than normal & $40(11.2)$ & $20(7.4)$ & $20(23.0)$ & $\mathbf{3 . 7 5}(1.91-7.36)$ \\
\hline Feel more irritable & $38(10.6)$ & $14(5.2)$ & $24(27.6)$ & $6.99(3.42-14.29)$ \\
\hline Feel more aggressive & $36(10.1)$ & $15(5.5)$ & $21(24.1)$ & $\mathbf{5 . 4 3}(2.65-11.11)$ \\
\hline Feel more nauseated & $36(10.1)$ & $19(7.0)$ & $17(19.5)$ & $3.22(1.59-6.52)$ \\
\hline $\begin{array}{l}\text { Find yourself unable to remember what you } \\
\text { did }\end{array}$ & $33(9.2)$ & $6(2.2)$ & $27(31.0)$ & $19.88(7.86-50.27)$ \\
\hline Feel more confused & $31(8.7)$ & $9(3.3)$ & $22(25.3)$ & $\mathbf{9 . 8 5}(4.33-22.41)$ \\
\hline $\begin{array}{l}\text { Experience a burning sensation in your eyes or } \\
\text { throat }\end{array}$ & $30(8.4)$ & $14(5.2)$ & $16(18.4)$ & $4.14(1.93-8.88)$ \\
\hline Develop chestpain & $28(7.8)$ & $13(4.8)$ & $15(17.2)$ & $4.13(1.88-9.09)$ \\
\hline Have slurred speech & $27(7.5)$ & $6(2.2)$ & $21(24.1)$ & $14.05(5.45-36.21)$ \\
\hline Have blurred vision & $27(7.5)$ & $7(2.6)$ & $20(23.0)$ & $\mathbf{1 1 . 2 6}(4.57-27.73)$ \\
\hline Feel more depressed & $22(6.1)$ & $8(3.0)$ & $14(16.1)$ & $6.30(2.55-15.61)$ \\
\hline Fear that you might be going crazy & $12(3.4)$ & $4(1.5)$ & $8(9.2)$ & $p<.001^{\mathrm{d}}$ \\
\hline Feel more sexually aroused & $11(3.1)$ & $1(0.4)$ & $10(1.2)$ & $p<.001^{\mathrm{d}}$ \\
\hline Feel more suicidal & $9(2.5)$ & $2(0.7)$ & $7(8.0)$ & $p<.001^{\mathrm{d}}$ \\
\hline Hear things that were not there & $8(2.2)$ & $2(0.7)$ & $6(6.9)$ & $p<.003^{\mathrm{d}}$ \\
\hline See things that were not there & $7(2.0)$ & $0(0.0)$ & $7(8.0)$ & $p<.001^{\mathrm{d}}$ \\
\hline
\end{tabular}

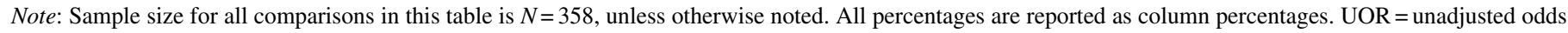
ratio. $\mathrm{CI}=$ confidence interval. Values in bold are statistically significant based on a 95\% CI that does not bound the value 1.0 .

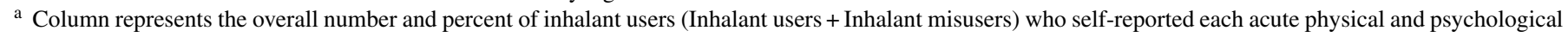
response.

b Inhaler users are the reference group for all unadjusted odds ratios.

c Sample size, $N=357$.

d Fisher's exact test used due to compute statistical significance due to cell counts less than 5. 
from inhaler use compared to inhaler users $(\mathrm{OR}=33.64,95 \%$ $\mathrm{CI}=14.82-76.33)$. Other significant effects included problems with memory $(\mathrm{OR}=19.88,95 \% \mathrm{CI}=7.86-50.27)$, slurred speech $(\mathrm{OR}=14.05,95 \% \mathrm{CI}=5.45-36.21)$, blurred vision $(\mathrm{OR}=11.26,95 \% \mathrm{CI}=4.57-27.73)$, and dizziness $(\mathrm{OR}=10.03$, $95 \% \mathrm{CI}=5.78-17.43)$.

\section{Discussion}

More than one-quarter (26\%) of youth surveyed in this study reported a lifetime diagnosis of asthma, a rate twice that of comparably aged youth in the general population (Akinbami, 2007). Most youth diagnosed with asthma (91.2\%) received a prescription for an inhaler. Given the widespread prevalence of asthma among youth in the U.S. and pervasive diffusion of asthma inhalers across many, if not most, population subgroups, it was not surprising that at least some proportion of the high-risk sample we studied reported asthma inhaler misuse. However, we found that $27 \%$ of youth who had been prescribed an asthma inhaler reported having intentionally used it excessively and that one-third of all youth in the sample had used an asthma inhaler without a prescription. Further, such use was frequently motivated by the intention to "get high" and associated with potentially serious health and social consequences. Although it is clear that a substantial proportion of youth engage in non-prescribed use of inhalers for the purposes of self-medication of asthma attacks (itself a potentially worrisome finding), it is equally evident that many youth were abusing inhalers for their psychoactive effects. Approximately one-in-eight youth in Missouri Division of Youth Services care at the time of the survey had used an asthma inhaler to get high.

Bivariate findings indicated that inhaler misusers tended to have an earlier onset of antisocial conduct, and higher levels of current psychiatric distress, temperamental fearlessness and impulsivity, and lifetime substance abuse problems, volatile solvent abuse and suicidality than did inhaler users. Each of these factors is a known correlate of substance use more generally. The results also showed that rates of asthma inhaler misuse were comparatively elevated among girls and whites in this sample. Caucasian racial status was a significant risk factor for inhaler misuse in multivariate logistic regression analyses confined to lifetime inhaler users/misusers. These demographic findings are consistent with those reported by Wu et al. (2004) and Howard et al. (2007) for volatile solvent abuse. Given that a history of volatile solvent inhalant use was the best discriminator of inhaler users and misusers in multivariate analyses, it may be appropriate to consider asthma inhaler misusers as a subgroup of inhalant users more generally.

Taken together, these findings indicate that asthma inhaler misuse is prevalent in the adolescent population we studied, frequently motivated by the desire to become intoxicated, and significantly associated with psychiatric dysfunction, substance abuse, and other correlates of these problems. Prior experience with and access to an inhaler appears to increase risk for inhaler misuse, as Boyd et al. (2006) also reported. Our findings indicate that inhaler misuse for the purposes of becoming intoxicated is both widespread and may justifiably be regarded as a form of substance abuse in many cases; thus, it is important to learn whether youth develop physical dependence on these agents, including tolerance and withdrawal symptoms, and what the short and long-term medical and social consequences of such abuse might be.

This study is the first to examine the nature and prevalence of various acute reactions to inhaler use/misuse among adolescent inhaler users and misusers. The inhaler misuser group experienced comparatively more numerous and serious reactions than did the inhaler user group. Adverse reactions to acute inhaler use were prevalent among inhaler misusers, with $62.1 \%$ feeling more dizzy during or immediately following inhaler use, $47.1 \%$ having a headache, $43.0 \%$ reporting rapid heart beat, $45.3 \%$ feeling more anxious, $33.3 \%$ feeling panicky, $33.3 \%$ feeling more tired or fatigued, $27.6 \%$ feeling more irritable, $25.3 \%$ feeling more confused, and $31.0 \%$ reporting memory problems. Positive reactions to inhaler use were also substantially more prevalent among inhaler misusers than users. Inhaler misusers were significant more likely than users to report experiences of euphoria (50.6\%), feeling relaxed $(40.2 \%)$, feeling more talkative $(37.9 \%)$, and feeling more powerful or confident $(23.0 \%)$ during or immediately following inhaler use. Little is currently known about the adverse consequences and perceived benefits of short- and longterm intentional inhaler misuse. This is clearly an area meriting more research attention in the future.

Although many of the findings reported above are unprecedented in the asthma or substance abuse literatures and important in their implications, several limitations characterize this research. Our survey findings were cross-sectional in nature and thereby preclude conclusions of a causal nature. The client population studied consisted of minimally-to-seriously antisocial youth; thus, findings may not generalize to youth in the general population. Further, the survey relied on self-report measures of asthma inhaler use and other variables. Given these limitations, we urge caution in interpreting and applying these findings. Strengths of the current investigation included the large sample, high respondent participation rate, under researched study population (i.e., antisocial youth in residential care), use of face-to-face interviews with structured assessments across a variety of measurement domains, and minimal missing data.

We would also advise future investigators in this area to include a more detailed assessment of asthma inhaler use. At present, asthmatic patients may receive corticosteroid and/or beta-2 agonist inhaler treatment; OTC asthma inhalers are also available for purchase. Although detailed assessment of type of inhaler used/misused would clearly have been preferable to our approach, we believe most of the responses to our survey items reflected reactions to use of albuterol inhalers, given the widespread current availability and use of this product in the community. Qualitative investigations might provide greater understanding as to the reasons why some youth misuse inhalers and better appreciation of the phenomenology of asthma inhaler intoxication.

Finally, it should be noted that the U.S. Food and Drug Administration has recently taken steps to remove albuterol inhalers with fluroniated hydrocarbon propellants from the marketplace by December, 2008 (Lavelle, 2007). More expensive 
albuterol inhalers will continue to be available with propellants such as hydrofluoroalkane. However many consumer advocates do not support removal of the relatively inexpensive generic albuterol inhalers with fluorocarbon propellants. It also remains unclear whether misuse and abuse of albuterol inhalers occurs because of the reinforcing properties of albuterol, the fluorocarbon propellants themselves, or a combination of the two agents. Additional research on asthma inhaler misuse and abuse will provide a substantially more solid foundation for policy making and clinical interventions in this area. At present, our findings suggest that misuse of asthma inhalers for their psychoactive effects may be prevalent and largely hidden in some patient and service populations. Large studies of asthma inhaler misuse in general youth and adult populations are needed to determine whether the findings reported herein generalize wholly, or in part, to the population more broadly.

\section{Conflict of interest}

Both authors declare they have no conflicts of interest.

\section{Acknowledgements}

Role of funding source: This study was supported by grants DA021405 (Natural History, Comorbid Mental Disorders, and Consequences of Inhalant Abuse, M.O. Howard, PI) and DA15929 (Neuropsychiatric Impairments in Adolescent Inhalant Users, M.O. Howard, PI) from the National Institute on Drug Abuse. The National Institute on Drug Abuse had no further role in study design; in the collection, analysis and interpretation of data; in the writing of the report; or in the decision to submit the paper for publication.

Contributors: The second author of this study designed the survey and supervised the collection of the data and assisted with statistical analyses. Both authors jointly conceptualized this study and prepared the manuscript. The authors would like to acknowledge Rachel Bates for her assistance with preparing the manuscript.

\section{References}

American Academy of Allergy Asthma \& Immunology, website accessed on August 01, 2008, material dated May 09, 2006: http://www.aaaai.org/aadmc/ ate/category.asp?cat=9893.

Akinbami, L., 2007. Asthma Prevalence, Health Care use and Mortality: United States, 2003-05. National Center for Health Statistics, Washington, DC.

Boyd, C.J., Teter, C.J., McCabe, S.E., 2004. Pilot study of abuse of asthma inhalers by middle and high school students. J. Adolesc. Health 34, 531-534.

Boyd, C.J., McCabe, S.E., Teter, C.J., 2006. Asthma inhaler misuse and substance abuse: a random survey of secondary school students. Addict. Behav. 31, 278-287.

Brennan, P.O., 1983. Addiction to aerosol treatment. Br. Med. J. (Clin. Res. Ed.) 287, 1877.

Derogatis, L.R., 1993. BSI Bibliography. National Computer Systems, Minneapolis, $\mathrm{MN}$.
Edwards, J.G., Holgate, S.T., 1979. Dependency upon salbutamol inhalers. Br. J. Psychiatry 134, 624-626.

Gaultier, M., Gervais, P., Lagier, G., Danan, L., 1976. Psychological pharmacodependency on salbutamol aerosol in an asthmatic. Therapie 31, 465-470.

Gluckman, L., 1974. Letter: ventolin psychosis. NZ Med. J. 80, 411.

Goodwin, R.D., Fischer, M.E., Goldberg, J., 2007. A twin study of post-traumatic stress disorder symptoms and asthma. Am. J. Respir. Crit. Care Med. 176, 983-987.

Grisso, T., Barnum, R., 2000. Massachusetts Youth Screening InstrumentSecond Version. User's Manual and Technical Reports. University of Massachusetts Medical School, Boston, MA.

Harrell, F.E., 2002. Hmisc and Design libraries. Available at: http://biostat.mc.vanderbilt.edu/twiki/bin/view/Main/RS. Accessed October 15, 2007

Howard, M.O., Jenson, J.M., 1999. Inhalant use among antisocial youth: prevalence and correlates. Addict. Behav. 24, 59-74.

Howard, M.O., Balster, R.L., Cottler, L.B., Wu, L.T., Vaughn, M.G., 2007. Inhalant use among incarcerated adolescents in the United States: prevalence, characteristics, and correlates of use. Drug Alcohol Depend. 93, 197-209.

IMS Intelligence, 2007. Retrieved September 6, 2007 from http://www.imshealth.com.

Katon, W., Lozano, P., Russo, J., McCauley, E., Richardson, L., Bush, T., 2007. The prevalence of DSM-IV anxiety and depressive disorders in youth with asthma compared with controls. J. Adolesc. Health 41, 455-463.

Kjellman, B., 1977. Case report: an asthmatic child with a very high consumption of ventoline respirator solution. Scand. J. Respir. Dis. Suppl. 101, 119-121.

Kuehn, B.M., 2008. Asthma linked to psychiatric disorders. JAMA 299, $158-160$.

Lavelle, M., 2007. Asthma's new expense: phaseout of inhaler leaves patients gasping with sticker shock. U.S. News \& World Report, March 19, 2007.

Lilienfeld, S.O., Andrews, B.P., 1996. Development and preliminary validation of a self-report measure of psychopathic personality traits in noncriminal populations. J. Pers. Assess. 66, 488-524.

Mannino, D.M., Homa, D.M., Akinbami, L.J., Moorman, J.E., Gwynn, C., Redd, S.C., 2002. Surveillance for asthma-United States, 1980-1999. MMWR Surveill Summ. 51, 1-13.

Martin, W., Unutzer, J., Szuba, M.P., 1995. Exacerbation of psychosis associated with inhaled albuterol. J. Clin. Psychopharmacol. 15, 446-447.

O'Callaghan, C., Milner, A.D., 1988. Aerosol treatment abuse. Arch. Dis. Child. 63,70 .

Prasher, V.P., Corbett, J.A., 1990. Aerosol addiction. Br. J. Psychiatry 157, 922-924.

Pratt, H.F., 1982. Abuse of salbutamol inhalers in young people. Clin. Allergy 12, 203-209.

Raine, J.M., 1984. Addiction to aerosol treatment. Br. Med. J. (Clin. Res. Ed.) 288, 241.

Rakhmanina, N.Y., Kearns, G.L., Farrar 3rd, H.C., 1998. Hypokalemia in an asthmatic child from abuse of albuterol metered dose inhaler. Pediatr. Emerg. Care 14, 145-147.

Rudd, R.A., Moorman, J.E., 2007. Asthma incidence: data from the National Health Interview Survey, 1980-1996. J. Asthma 44, 65-70.

Schnapf, B.M., Santeiro, M.L., 1994. Beta-agonist inhaler causing hallucinations. Pediatr. Emerg. Care 10, 87-88.

Slessor, I.M., 1984. Addiction to aerosol treatment. Br. Med. J. (Clin. Res. Ed.) 288,485

Thompson, P.J., Dhillon, P., Cole, P., 1983. Addiction to aerosol treatment: the asthmatic alternative to glue sniffing. Br. Med. J. (Clin. Res. Ed.) 287, 1515-1516.

Wu, L.T., Pilowsky, D.J., Schlenger, W.E., 2004. Inhalant abuse and dependence among adolescents in the United States. J. Am. Acad. Child Adolesc. Psychiatry 43, 1206-1214. 